\title{
NONCOMMUTATIVITY, SUPERSYMMETRY BREAKING, AND MODEL BUILDING
}

\author{
GABRIELE TRAVAGLINI \\ Department of Physics and IPPP, University of Durham \\ Durham DH1 3LE, UK \\ E-mail: gabriele.travaglini@durham.ac.uk
}

\begin{abstract}
We argue that in the presence of supersymmetry, an ordinary commutative $S U(N)$ gauge theory with a general matter content can always be embedded into a noncommutative $U(N)$ theory at energies above the noncommutativity mass scale. The IR/UV mixing crucially decouples the $U(1)$ degrees of freedom in the infrared. We also discuss the special rôle of the $U(1)$ degrees of freedom in triggering the dynamical breaking of supersymmetry in a noncommutative framework, and outline how noncommutative Grand Unification might be achieved.
\end{abstract}

Noncommutative field theories are a rich subject with a variety of unexpected and intriguing features. ${ }^{\text {a }}$ The notion of the smearing of the space-time structure at distances smaller than the Planck length seems to be a natural consequence of the basic principles of Quantum Mechanics and General Relativity; early considerations in this direction date back, not surprisingly, to Dirac and Heisenberg. More recently, the importance of theories on noncommutative backgrounds has been emphasised by many recent developments in string and field theory ${ }^{3,4,5}$. $\mathrm{In}^{5}$, Seiberg and Witten have shown that space-time noncommutativity, $\left[x^{\mu}, x^{\nu}\right]=i \theta^{\mu \nu}$, naturally arises in the low-energy description of open strings ending on D-branes in the presence of a constant $B$-field. A noncommutative field theory can be equivalently reformulated as a deformation of an ordinary quantum field theory in which the product of fields is replaced by an associative, noncommutative product (the so-called "star" or "Moyal" product) ${ }^{6}$,

$$
(f \star g)(x) \equiv f(x) \exp \left(\frac{i}{2} \theta^{\mu \nu} \stackrel{\leftarrow}{\partial_{\mu}} \overrightarrow{\partial_{\nu}}\right) g(x)=f(x) g(x)+\frac{i}{2} \theta^{\mu \nu} \partial_{\mu} f \partial_{\nu} g+\cdots
$$

This observation has far-reaching consequences: it leads to the possibility of analyzing their dynamics with the standard, powerful field theory techniques based on functional integration. The combined effect of an uncertainty principle on space-time coordinates with the usual Heisenberg principle leads to a curious mixing of ultraviolet and infrared effects, which in some cases generates infrared singularities in the low energy effective action. It has been shown that this infrared/ultraviolet (IR/UV) mixing ${ }^{7,8}$ dramatically invalidates the naive expectation about a universal behaviour in the infrared for commutative and noncommutative theories: namely, the infrared regime of a noncommutative theory is in general completely different from that of its commutative counterpart and, most importantly, a noncommutative gauge theory must be supersymmetric in order to be consistent $^{7,8,9}$. As an example, consider a noncommutative Super Yang-Mills theory with

\footnotetext{
${ }^{\mathrm{a}}$ For recent reviews and extensive lists of references see ${ }^{1,2}$.
} 
gauge group $U(N)$ (theories based on $S U(N)$ are not consistent). The leading order terms in the derivative expansion of the Wilsonian effective action read ${ }^{9,10}$ :

$$
\mathcal{L}_{\text {eff }}=-\frac{1}{4 g_{1}^{2}(k)} F_{\mu \nu}^{U(1)} F_{\mu \nu}^{U(1)}-\frac{1}{4 g_{N}^{2}(k)} F_{\mu \nu}^{S U(N)} F_{\mu \nu}^{S U(N)}+\cdots,
$$

where the dots stand for terms involving fermions and higher-derivative corrections. The multiplicative coefficients in front of the gauge kinetic terms in (2) define the Wilsonian coupling constants of the corresponding gauge factors. Their dependence on the momentum scale $k$ is displayed in Figure 1 . The running of the $U(1)$ has the following asymptotic behaviour:

$$
\frac{1}{g_{1}^{2}(k)} \rightarrow \pm \frac{3 N}{(4 \pi)^{2}} \log k^{2},
$$

where the plus (minus) sign corresponds to $k^{2} \rightarrow \infty\left(k^{2} \rightarrow 0\right)$, whereas for the $S U(N)$ gauge factor we have, in both limits:

$$
\frac{1}{g_{N}^{2}(k)} \rightarrow \frac{3 N}{(4 \pi)^{2}} \log k^{2}
$$

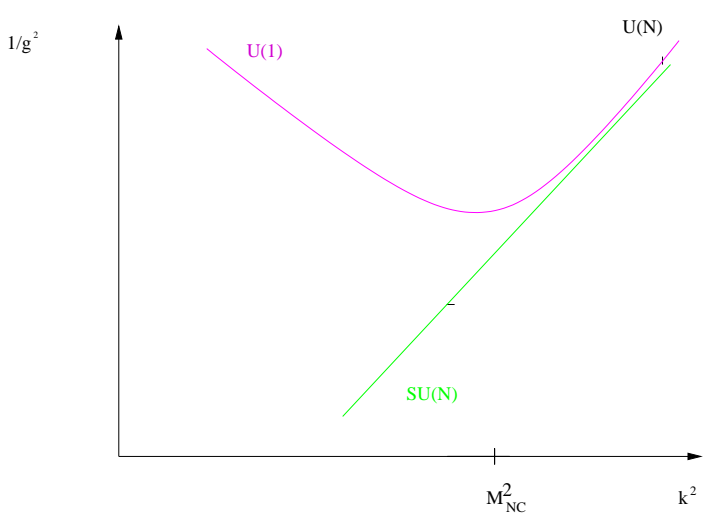

Figure 1. Running of the effective couplings as a function of the Wilsonian scale $k$.

The change in the running of the $U(1)$ coupling in (3) occurs at the scale $k^{2} \sim M_{N C}^{2}$, where $M_{N C} \sim \theta^{-1 / 2}$ is the noncommutative mass. This running was interpreted in ${ }^{11}$ as having a full noncommutative $U(N)$ gauge theory in the UV, which in the low-energy limit appears as a commutative $S U(N)$ theory, with the $U(1)$ degrees of freedom which become progressively more weakly coupled (i.e. unobservable) in the infrared. Note that this does not implies that the noncommutative $U(N)$ gauge symmetry is broken. In fact, a gaugeinvariant completion of (2) was proposed in ${ }^{12,13}$ which involves open Wilson lines. This completion introduces higher-derivative terms, which are irrelevant for the low-energy dynamics.

The question then arises as to whether it is possible to find a realistic, nonsupersymmetric theory for Particle Physics based on a noncommutative set-up: the issue of supersymmetry breaking is clearly crucial. We found that, remarkably, noncommutative supersymmetric gauge theories solve this problem in a self-consistent way. ${ }^{11}$ The general mechanism works as follows. As reminded above, noncommutative gauge theories contain a $U(1)$ factor; supersymmetry gets broken in the $U(1)$ sector, which gets decoupled in the infrared theory. This supersymmetry breaking can be easily accomplished by 
adding to the tree level Lagrangian a Fayet-Iliopoulos D-term, $\mathcal{L}_{F I}=\xi_{F I} \int d^{2} \theta d^{2} \bar{\theta} \operatorname{tr}_{N} V$, where $V$ is the real $U(N)$ vector superfield and the trace over the $N$ by $N$ matrices selects the $U(1)$-component of $V$. The Fayet-Iliopoulos action, $\int d^{4} x \mathcal{L}_{F I}$, is invariant under the noncommutative $U(N)$ group, and can be naturally introduced in any $U(N)$ theory. This provides us with a scenario of a "gauge-mediated" supersymmetry breaking, where the $U(1)$ degrees of freedom, which eventually become arbitrarily weakly coupled in the IR, play the rôle of the hidden sector ${ }^{11}$. Both the hidden sector and the messenger sector are naturally part of the noncommutative $U(N)$ gauge theory. We would like to stress that this mechanism of dynamical supersymmetry breaking is deeply intertwined with the IR/UV mixing, which affects only the $U(1)$ degrees of freedom.

Finally, in ${ }^{14}$ we were able to construct general representations of noncommutative $U(N)$ groups, which are needed in order to apply these ideas to more general contexts, as Grand Unified Theories.

We conclude with a few comments. The results of our analysis show that in the presence of supersymmetry, an ordinary commutative $S U(N)$ gauge theory with a general (anomaly free) matter content can always be embedded into a noncommutative $U(N)$ theory at energies above the noncommutativity mass scale. At energies below this scale, the $U(1)$ degrees of freedom decouple due to the infrared/ultraviolet mixing, and the noncommutative theory reduces to its commutative counterpart. The mixing of the ultraviolet with the infrared is the source of very unusual phenomena in field theory, and leads to conjecture novel scenarios of dynamical supersymmetry breaking. Moreover, the possibility of constructing generic-rank representations of noncommutative groups opens a new avenue of research, focusing on formulating noncommutative extensions of Grand Unified Theories. This makes it more plausible that a noncommutative set-up might be relevant for a unified description of interactions.

We have not addressed the issue of anomaly (non-)cancellation in noncommutative chiral theories. This is an important point, which deserves to be carefully studied in order to construct a proper noncommutative version of the (supersymmetric) Standard Model.

The challenge we face is, therefore, to construct realistic models for Particle Physics based on a noncommutative formulation.

It is a pleasure to thank Chong-Sun Chu and Valya Khoze for a very enjoyable and stimulating collaboration on the subject of this talk. We would also like to thank Nick Dorey, Tim Hollowood, Tim Jones, Gordy Kane, Prem Kumar, Gian Carlo Rossi and Massimo Testa for discussions.

\section{References}

1. M. R. Douglas and N. A. Nekrasov, Rev. Mod. Phys. 73 (2001) 977 [hep-th/0106048].

2. L. Castellani, Class. Quant. Grav. 17 (2000) 3377 [hep-th/0005210].

3. A. Connes, M. R. Douglas, and A. Schwarz, JHEP 9802 (1998) 003 [hep-th/9711162].

4. M. R. Douglas and C. M. Hull, JHEP 9802 (1998) 008 [hep-th/9711165].

5. N. Seiberg and E. Witten, JHEP 9909 (1999) 032 [hep-th/9908142].

6. S. Doplicher, K. Fredenhagen ,and J. E. Roberts, Phys. Lett. B 331 (1994) 39.

7. S. Minwalla, M. Van Raamsdonk, and N. Seiberg, JHEP 0002 (2000) 020 [hep-th/9912072]. 
8. A. Matusis, L. Susskind, and N. Toumbas, JHEP 0012 (2000) 002 [hep-th/0002075].

9. V. V. Khoze and G. Travaglini, JHEP 0101 (2001) 026 [hep-th/0011218].

10. T. J. Hollowood, V. V. Khoze, and G. Travaglini, JHEP 0105 (2001) 051 [hep-th/0102045].

11. C. S. Chu, V. V. Khoze, and G. Travaglini, Phys. Lett. B 513 (2001) 200 [hep-th/0105187].

12. M. Van Raamsdonk, JHEP 0111 (2001) 006 [hep-th/0110093] .

13. A. Armoni and E. Lopez, Nucl. Phys. B 632 (2002) 240 [hep-th/0110113] .

14. C. S. Chu, V. V. Khoze, and G. Travaglini, Phys. Lett. B 543 (2002) 318 [hep-th/0112139]. 